\title{
Standard Underpotential Deposition Shift. A new parameter to characterize the UPD phenomenon.
}

Rafael A. Vicente, Heloisa V. N. Gomes, Pablo S. Fernándezz .

Chemistry Institute, University of Campinas, PO Box 6154, 13083-970, Campinas SP, Brazil.

zpablosf@unicamp.br

\begin{abstract}
Underpotential deposition (UPD) is a phenomenon where atoms of an element $\mathrm{M}$ are deposited from ions $\mathrm{M}^{\mathrm{n}+}$ on a substrate $\mathrm{S}$ at potentials more positive than for the deposition of $\mathrm{M}^{\mathrm{n}+}$ on $\mathrm{M}$. These systems have been studied for more than a century and are interesting from both the applied and the fundamental point of view. Despite the vast literature on the subject, there is no thermodynamic parameter so far able to characterize an UPD system. Even if the so-called "UPD shift" has been used for decades, the limitations of this parameter has been fully recognized in the field. Herein, using a simple Nernstian treatment and straightforward measurements, we show how to measure and calculate a new proposed fundamental thermodynamic parameter namely, the "Standard UPD potential" $\left(E_{M^{n+} / M(S)}^{0}\right)$. We showed results for the deposition of $\mathrm{Cu}^{2+}$ on $\mathrm{Au}$ in acidic media, in solutions containing $\mathrm{ClO}_{4}^{-}$or $\mathrm{HSO}_{4}^{-} / \mathrm{SO}_{4}^{2-}$ anions. We obtained $E_{\mathrm{Cu}}^{0+} / \mathrm{Cu}(\mathrm{Au})=0.65 \pm 0.02 \mathrm{~V}$, independently of the concentration of the acid and the nature of the anion.
\end{abstract}

\section{Introduction}

Underpotential deposition (UPD) is a term that refers to the deposition of a foreign atom (M) on a substrate (S) at positives electrochemical potential with respect to the potential for the massive deposition of $\mathrm{M}\left(E_{M^{n+} / M}\right)$. Thus, the deposition occurs at positive overpotentials (underpotential is an unfortunate denomination) with respect to $E_{M^{n+} / M}$.

Besides an originally surprising and interesting phenomenon in physical chemistry, the quantity of published articles involving UPD has increased continuously during the last 5 decades due to its application in several important fields of chemistry. UPD of metals is an important method for the electrochemical preparation of catalysts ${ }^{1,2}$ and sensors ${ }^{3,4}$. It is also used in the preparation of semiconductors ${ }^{5-7}$ and controlled metal alloys ${ }^{8}$. Moreover, it is widely used for quantification of active sites (or electroactive areas) in noble metal-based catalysts $^{9-11}$. It is surprising that, despite the importance of the topic, there is only one book, published in 2015 by Leiva et al., exclusively devoted to discuss both fundamental and experimental aspects of the UPD ${ }^{12}$. Although UPD is usually limited to the deposition of a less noble metal on a more noble substrate, some important contributions showed that it can also be used to deposit noble metals such as $\mathrm{Pt}$ and $\mathrm{Pd}^{13,14}$. 
In this introduction we will focus only on some pioneering works in the field and more recent contributions that allow us to show the importance of the proposed thermodynamic parameter. Thus, we refer the readers to the vast literature, starting by the excellent book mentioned earlier, for details about both the experimental and theoretical developments in the field.

UPD was observed for the first time in the late $19^{\text {th }}$ century with the raise of radiochemistry by Moïse Haïssinky during early studies of the electrochemistry of radioisotopes ${ }^{15}$, but it was in 1974 when Gerischer, Kolb and Przasnyski published two papers ${ }^{16,17}$ giving the first phenomenological explanation for UPD. They argued that the UPD shift $\left(\triangle E^{U P D}\right)$, i.e., the difference between the potential for the equilibrium of bulk deposition/oxidation $\left(E_{M^{n+} / M}\right)$ and the potential for the desorption of the foreign atom monolayer $\left(\Delta E^{U P D}\right)$, changes linearly with the difference between the work functions of M and S. In 1975, Prof. Trasatti, using the same data, improved the correlation by considering the onset of the UPD ${ }^{18}$.

Most (if not all) the papers published in the field in the 70s were performed using polycrystalline electrodes. Works using single crystal electrodes revealed the complexity of $\mathrm{UPD}^{19}$, providing voltammograms showing multiple peaks that made it impossible to use the original definition of $\triangle E^{U P D}$. These uncertainties are not surprising, as the definition and calculation of $\triangle E^{U P D}$ carries serious oversimplification of the problem. In fact, the experimental values were obtained at arbitrary concentrations of $M^{n+}$, using different electrolytes, using arbitrary concentrations of the electrolyte and usually using voltammetry at relatively high scan rates, measuring in conditions far from equilibria ${ }^{16,17}$.

Despite the serious inconsistencies in the definition/calculation of $\triangle E^{U P D}$, it remains as the only parameter used to "characterize" an UPD system. In 2017, Weitzner and Dabo ${ }^{20}$, using a quantum-continuum model of the electrochemical interface, studied the influence of the electrochemical potential, concentration of ions, adsorbate coverage and anion adsorption effect on the UPD of $\mathrm{Cu}$ on $\mathrm{Au}(100)$. This study paves the way for more systematic studies in the area considering a more realistic system in comparison to computational experiments in vacuum. Unfortunately, the authors used $\triangle E^{U P D}$ as the experimental result to be compared to the computational ones. Apart from this excellent contribution, it is worth noting that several pages of the book published by Leiva et. al. ${ }^{12}$ were devoted to discussing concepts closely related to $\triangle E^{U P D}$. Thus, the aim of this study is to set the bases for the calculation and measurement of a new parameter able to provide a more reliable thermodynamic quantity to characterize UPD systems. It is important to note that this parameter will not describe the UPD behaviour in the complete potential domain, once that it is an extremely complex process whose mathematical treatment would need to consider several factors.

Herein, we tackle the problem in the frame of the Nernst formalism. We propose a new UPD shift, the standard UPD shift $\left(\triangle E_{U P D}^{0}\right)$, which we define as the difference between the standard potential for bulk deposition $\left(E_{M^{n+} / M}^{0}\right)$ and standard potential of UPD $\left(E_{M^{n+} / M(S)}^{0}\right)$. This quantity is far more fundamental than the one proposed by Kolb et al. ${ }^{16,17}$, as it is independent of the electrons exchanged in the reaction, of the activity of the cation and of the electrode 
coverage. Finally, to show how to calculate $\triangle E_{U P D}^{0}$ and to obtain the first value for this parameter, we revisited the UPD of $\mathrm{Cu}$ on $\mathrm{Au}_{\mathrm{p}}$ (polycrystalline $\mathrm{Au}$ ) in the presence of $\mathrm{ClO}_{4}^{-}$ and $\mathrm{HSO}_{4}^{-} / \mathrm{SO}_{4}^{2-}$.

\section{Experimental}

\section{Electrochemical system.}

A standard three-electrode cell and potentiostat/galvanostat (Autolab PGSTAT302N, Methrom ${ }^{\circledR}$ ) were used in all the experiments, which were performed under temperature control using a thermostatic bath (MQ8TC 99-20, Microquímica®) set in $298 \mathrm{~K}$.

For the electrochemical experiments, the working electrode (WE) consisted of a $\mathrm{Au}_{\mathrm{p}}$ wire with a spherical ending. The working electrode was prepared by using a gold wire $(0.05 \mathrm{~mm}$ diameter, 99,95\%) that was carefully melted until forming spontaneously a sphere on its end (with a diameter of about $1 \mathrm{~mm}$ and electrochemical active area of $0.235 \mathrm{~cm}^{2}$ ). A Aup coil was used as counter electrode (CE) and the reference electrode (RE) was a reversible hydrogen electrode (RHE). All potentials mentioned in this work refer to the RHE or SHE. The potentials measured vs. RHE were converted to the SHE scale using the Nernst equation and the activity coefficients extracted from the literature ${ }^{21-23}$.

Between each experiment, WE and CE were cleaned by immersion in concentrated nitric acid for about $10 \mathrm{~s}$, then flame annealed until the electrode started to glow red, and finally quenched and rinsed with ultrapure water. For the working electrode, after growing the sphere, instead of quenching with water, this procedure was repeated several times quenching the electrode with nitric acid. This procedure has proven to be important for wires bought from different companies, with different purities and it is the approach we used to generate highly pure spheres for building single crystal electrodes using the well-known Clavilier's method ${ }^{24}$. Then the WE was cleaned electrochemically by fast cycling between -0.5 to $2.0 \mathrm{~V}$. Before the UPD measurements, we performed blank voltammograms to verify the electrode surface, which presented the expected profile reproducibly.

The RE was also flame annealed and rinsed with ultrapure water $\left(18.2 \mathrm{M} \Omega . \mathrm{cm}^{-1}, 25^{\circ} \mathrm{C}\right.$, Millipore). The oxygen was removed from the cell bubbling Argon 5.0 for at least 10 minutes before cycling. During the cycling, an Argon atmosphere was maintained with a continuous flux over the solution inside the cell to prevent oxygen entrance.

Open circuit potential (OCP) experiments consisted in measuring the potential difference between a $\mathrm{Cu}_{\mathrm{p}}$ wire and the RHE. The $\mathrm{Cu}_{\mathrm{p}}$ was cleaned by quick immersion in concentrated nitric acid followed by rinsing with ultrapure water.

\section{Chemicals.}

All solutions were made with ultrapure water, and the chemicals were used without any prior purification. The chemicals used were sulfuric acid (ISO grade, Merck Emsure®), perchloric acid (ISO grade, Merck Emsure ${ }^{\circledR}$ ), copper (II) sulfate pentahydrate (Sigma-Aldich ${ }^{\circledR}$ 
$\geq 98 \%$, ReagentPlus ${ }^{\circledR}$ grade), copper perchlorate hexahydrate (Sigma-Aldrich $\AA$, reagent grade) and nitric acid (P.A., ACS, 65\%, Synth®).

\section{Theoretical considerations}

\section{UPD shift: a Nernstian treatment.}

Several theories were developed in the last 50 years to describe UPD systems. For details we refer the reader to the literature ${ }^{12}$. Here, we will employ a simple treatment based on the Nernst equation. By simple. we mean that we wrote the simplest possible reactions, i.e., involving only $M^{n+}, M$ and $S$.

$$
M^{n+}+n e^{-} \rightleftarrows M
$$

The equilibrium potential for the deposition of an atom $\mathrm{M}$ over a substrate also formed by atoms of $\mathrm{M}$ is given by:

$$
E_{M^{n+} / M}=E_{M^{n+} / M}^{0}+\frac{R T}{z_{b} F} \ln \left(\frac{a_{M^{n+}}}{a_{M}}\right)
$$

Where $E_{M^{n+} / M}$ is the equilibrium potential for the redox couple $M^{n+} / M, a_{M^{n+}}$ is the activity of the ions $M^{n+}, \mathrm{z}$ is the electrosorption valence (which is denoted by $\mathrm{z}_{\mathrm{b}}$ for the bulk deposition, and $\mathrm{z}_{\mathrm{u}}$ for the UPD), and $a_{M}=1$ and $n$ is the number of electrons exchanged in $\mathrm{R} 1$ (which is not always equal to the charge of the ion, but we decided to keep the discussion as simple as possible in this section).

Considering the molality scale, we get:

$$
E_{M^{n+} / M}=E_{M^{n+} / M(b u l k)}^{0}+\frac{R T}{z_{b} F} \ln \left(\gamma_{M^{n+}} m_{M^{n+}} / m^{0}\right)
$$

Where $\gamma_{M^{n+}}$ is the molality-scale activity coefficient for $M^{n+}$ and $m^{0}=1 \mathrm{~mol} \cdot \mathrm{kg}^{-1}$ (we will omit this term from now on for the sake of clarity). Thus, $E_{M^{n+} / M}^{0}$ is a fictitious state with $\gamma_{M^{n+}} m_{M^{n+}} / m^{0}=1$, i.e., we pretend ideally dilute behavior $\left(\gamma_{M^{n+}}=1\right)$ for $m_{M^{n+}} / m^{0}=1$. Thus, the $E_{M^{n+} / M}^{0}$ defined here is the standard potential for the equilibrium between redox couples widely used in chemistry and that can be found in the well-known "Electrochemical series tables" and accepted by IUPAC ${ }^{25}$.

For the deposition of a foreign metal $M$ over a substrate S (UPD), we can write:

$$
\begin{gathered}
M^{n+}+n e^{-} \rightleftarrows M(S) \quad(\mathrm{R} 2) \\
E_{M^{n+} / M(S)}=E_{M^{n+} / M(S)}^{0}-\frac{R T}{z_{u} F} \ln \left(\frac{a \theta_{M}}{a_{M^{n+}}}\right)
\end{gathered}
$$


Where $E_{M^{n+} / M(S)}$ is the equilibrium potential for the electroadsorption/desorption of M on $\mathrm{S}$ from a solution containing ions $M^{n+}$ with activity $a_{M^{n+}} a \theta_{M}$ is the activity of the atoms of $\mathrm{M}$ at the surface of the substrate and $n$ is the number of electrons exchanged in R2.

To relate $a \theta_{M}$ to a measurable quantity, we considered a Langmuir isotherm ${ }^{26}$. Substituting it into equation 3 we get an equation dependent on the electrode coverage, $\theta_{M}$.

$$
E_{M^{n+} / M(S)}=E_{M^{n+} / M(S)}^{0} \quad-\frac{R T}{z_{u} F} \ln \left(\frac{\theta_{M}}{\left(1-\theta_{M}\right) a_{M^{n+}}}\right)
$$

For low coverages $\theta_{M} \ll 1$ and $\frac{\theta_{M}}{1-\theta_{M}} \approx \theta_{M}$. Then:

$$
E_{M^{n+} / M(S)}=E_{M^{n+} / M(S)}^{0}-\frac{R T}{z_{u} F} \ln \left(\frac{\theta_{M}}{a_{M^{n+}}}\right)
$$

Considering the molality scale, we get:

$$
E_{M^{n+} / M(S)}=E_{M^{n+} / M(S)}^{0} \quad-\frac{R T}{z_{u} F} \ln \left(\frac{\theta_{M}}{\gamma_{M^{n+}} m_{M^{n+}} / m^{0}}\right)
$$

Where the term $\gamma_{M^{n+}} m_{M^{n+}} / m^{0}$ is the expanded metal ion activity term. Therefore, we define $E_{M^{n+} / S}^{0}$ as the fictitious state with: i) $\gamma_{M^{n+}} m_{M^{n+}} / m^{0}=1$ (in a similar way than $E_{M^{n+} / M}^{0}$ ) and ii) $\theta_{M}=1$. Thus, we pretend to observe the same behaviour of the systems at $\theta_{M}=1$ than when $\theta_{M} \rightarrow 0$, i.e., we also consider an ideally dilute behaviour at the surface.

We then define $\triangle E_{U P D}^{0}$ as the difference between the standard potential for the massive deposition and for the UPD.

$$
\Delta E_{U P D}^{0}=E_{M^{n+} / M}^{0}-E_{M^{n+} / M(S)}^{0}
$$

Hence, $\triangle E_{U P D}^{0}$ measures the difference in the standard potentials for $M^{n+} / M$ and for $M^{n+} / M(S)$ at $\theta_{M}=1$. These are two fictitious states where the $M^{n+}$ behaves as in an ideally dilute solution at $m_{M^{n+}}=1 \mathrm{~mol} \cdot \mathrm{kg}^{-1}$ and the adsorbates behaves at $\theta_{M}=1$ as at $\theta_{M} \rightarrow 0$.

This quantity is independent of the electrons exchanged in both processes, the activity of the cation and of the coverage as the adsorbates do not interact in standard state conditions. It is worth noting that the interaction between adatoms was also avoided in the paper of Trasatti ${ }^{18}$ by considering the potential for the onset of the UPD, but, unfortunately, working in nonstandard conditions, i.e., keeping some of the arbitrariness mentioned before. 
UPD shift: the measurement.

To obtain $\triangle E_{U P D}^{0}$ we need to know two quantities, namely, $E_{M^{n+} / M}^{0}$ and $E_{M^{n+} / M(S)}^{0}$. The former is the well-known standard potential for the equilibrium between $M^{n+} / M$ (several values are tabulated in the so-called "Electrochemical series tables") ${ }^{27}$. The latter can be measured as follows.

From equation 1, we have:

$$
E_{M^{n+} / M}=E_{M^{n+} / M}^{0}+\frac{R T}{z_{b} F} \ln \left(a_{M^{n+}}\right)
$$

If we know $E_{M^{n+} / M}^{0}$, measuring $E_{M^{n+} / M}$, we get the metal ions activity. The value of the activity can be inserted in equation 5 , which is only valid for $\theta_{M} \rightarrow 0$. Then, plotting $E_{M^{n+} / M(S)}$ vs. $\ln \left(\theta_{M} / a_{M^{n+}}\right)$, and fitting the results with an straight line for low coverages, we get an slope equal to $\quad-R T / z_{u} F$ and an intercept equal to $E_{M^{n+} / M(S)}^{0}$, which is the desired value. Finally, we get the UPD shift from equation (7). Please, note that some of the considerations in this last paragraph are similar to the discussions that can be found in any classical textbook of Physical-Chemistry about the Henry's Law and their use to calculate the vapor partial pressure of a solute above an ideally dilute solution and then to calculate its activity and activity coefficients in a real solution.

\section{Results}

Figure 1 shows the voltammograms for the UPD of $\mathrm{Cu}^{+2}$ on $\mathrm{Au}_{\mathrm{p}}$ in sulfuric and perchloric acid. The complete set of results are shown in Figures $\mathrm{S} 1$. We used concentrations of $\mathrm{Cu}^{2+}$ in solution ranging from $5 \times 10^{-5}$ to $1 \times 10^{-2} \mathrm{~mol} / \mathrm{kg}$ to be able to extrapolate the data to $a_{c u^{+2}}=1$ when needed.

The profiles are similar to those found in the literature, where the UPD of $\mathrm{Cu}$ ions on $\mathrm{Au}$ electrodes have been revisited many times along the last decades both, using pure electrochemical experiments ${ }^{28,29}$ and multi-technique approaches ${ }^{30-35}$. Thus, valuable information was obtained through experiments involving single crystal electrodes in acid media using X-rays absorption and diffraction techniques in synchrotron facilities and Scanning Tunnelling Microscopy in situ. Even if it is not the aim of our work to discuss the Cu structures on the $\mathrm{Au}_{\mathrm{p}}$ electrode, it is worth noting that the literature show that the deposition strongly depends on the Au crystalline plane ${ }^{29}$ and also of the composition of the electrolyte ${ }^{36-38}$. These facts explain the difference in the CV profiles shown in Figures 1 and S1. Besides, the intrinsic complexity of a polycrystalline electrode ${ }^{39}$ necessarily generates electrochemical responses coming from several parallel processes occurring simultaneously at the different facets and defects of the surface. 
For most of the experimental conditions, the $\mathrm{CV}$ profiles are asymmetric in the region of the bulk deposition in both electrolytes, indicating a lack of reversibility. The region for the bulk is poorly defined in $\mathrm{HClO}_{4}$ acid due to kinetic limitations. Avc1 et. al. ${ }^{40}$ showed that it can take more than an hour (depending on the conditions) to form a $\mathrm{Cu}$ monolayer on $\mathrm{Au}(111)$ at constant potential in $\mathrm{HClO}_{4}$. Thus, even at low scan rates, the $\mathrm{CV}$ profiles shows kinetic contributions, not allowing to apply a Nernstian treatment and, in consequence, obtain reliable values of $E_{\mathrm{Cu}^{2+} / \mathrm{Cu}}$. Consequently, to perform the measurement in conditions of electrochemical equilibrium, $E_{\mathrm{Cu}^{2+} / \mathrm{Cu}}$ was determined by measuring the OCP between a $\mathrm{Cu}_{\mathrm{p}}$ wire and the RHE in both electrolytes. On the other hand, the results show a much higher reversibility at the early stages of the UPD (low coverages) where the forward and backward scans look similar.

To find $\Delta E_{U P D}^{0}$, we need to determine the values of $a_{C u^{2+}}$ for each system studied in this work. Thus, we used $E_{\mathrm{Cu}^{2+} / \mathrm{Cu}}$ measurements to find the activities using equation 8. Table S1 shows the $E_{\mathrm{Cu}^{2+} / \mathrm{Cu}}$ values, which were determined by the OCP method. The activities were then calculated using $E_{\mathrm{Cu}^{2+} / \mathrm{Cu}}$ and the well-known value for $E_{\mathrm{Cu}^{2+} / \mathrm{Cu}}^{0}=0.339 \mathrm{~V}^{41-43}$ (Table S2).

The plot of $E_{\mathrm{Cu}^{2+} / \mathrm{Cu}}$ vs. $\ln \left(m_{\mathrm{Cu}^{2+}}\right)$ yields an excellent linear fit (Figure S3), which confirms the expected Nernstian behavior for the $\mathrm{Cu}^{2+} / \mathrm{Cu}^{0}$ equilibrium with $\mathrm{Zb} \approx 2$, validating our OCP measurements.

Figure 2 shows the plot $E_{\mathrm{Cu}^{2+} / \mathrm{Cu}}$ vs. $\ln \left(a_{\mathrm{Cu}^{2+}}\right)$ using $E_{\mathrm{Cu}^{2+} / \mathrm{Cu}}$. The slope of curve obtained using both molality and activities provides $\mathrm{z}_{\mathrm{b}}$ of approximately 2 , indicating the full discharge of the $\mathrm{Cu}^{2+}$ to form $\mathrm{Cu}^{0}$.

Next, we need to calculate $E_{C u^{2+} / C u(A u)}^{0}$. For that we extracted the $\mathrm{Cu}$ coverages from the UPD onset region and extrapolated the behavior to $\theta_{M}=1$ as discussed in the theoretical considerations section.

Figure 3 shows the plot of $E_{C u^{2+} / C u(A u)}$ vs. $\ln \left(\theta_{C u} / a_{C u^{2+}}\right.$ ) for low coverages (up to 0.05). We define coverage as $\theta_{C u}=Q_{\theta} / Q_{T}$, where $Q_{\theta}$ is the charge recorded at a given electrochemical potential and $Q_{T}$ is the total UPD charge (Figure S2).

Prior to the UPD onset region, the current comes from the region where the currents are mainly related to the double-layer charge/discharge. After the subtraction, the coverages calculated in this potential domain are extremely low and sensitive to the baseline, yielding a non-reproducible and non-linear $\ln \left(\theta_{C u} / a_{C u^{2+}}\right)$ vs. potential behaviour. On the other hand, after the UPD, the current sets in (as it can be clearly noticed from the voltammogram on the inset, around $0.7 \mathrm{~V}$ ) and we can fit the results with a straight line and extract values of $\mathrm{z}_{\mathrm{u}}$ and $E_{C u^{2+} / C u(A u)}^{0}$ vs. SHE (Table 1), by using equation 5. Table S3 and S4 show the complete set of results. 
Table 1. $\mathrm{Z}_{\mathrm{u}}$ and $E_{C u^{2+} / \mathrm{Cu}(\mathrm{Au})}^{0}$ mean values.

\begin{tabular}{|c|c|c|c|}
\cline { 2 - 4 } \multicolumn{1}{c|}{} & $\begin{array}{c}\mathrm{H}_{2} \mathrm{SO}_{4} \mathbf{0 . 0 5} \\
\mathbf{m o l} / \mathbf{k g}\end{array}$ & $\begin{array}{c}\mathrm{H}_{2} \mathrm{SO}_{4} \mathbf{0 . 5} \\
\mathbf{m o l} / \mathbf{k g}\end{array}$ & $\begin{array}{c}\mathrm{HClO}_{4} \mathbf{0 . 0 5} \\
\mathbf{m o l} / \mathbf{k g}\end{array}$ \\
\hline $\mathrm{Z}_{\mathbf{u}}$ & $1.5 \pm 0.2$ & $1.6 \pm 0.1$ & $1.7 \pm 0.1$ \\
\hline $\boldsymbol{E}_{M^{\boldsymbol{n}+} /(\boldsymbol{S})}^{\mathbf{0}}(\mathbf{V})$ & $0.65 \pm 0.02$ & $0.65 \pm 0.02$ & $0.64 \pm 0.01$ \\
\hline
\end{tabular}

Thus, we got $\mathrm{z}_{\mathrm{u}} \approx 1.6$ for the UPD in these electrolytes (considering the standard deviation, the values for all electrolytes were the same). The values lower than 2 suggest that: i) the ions are not completely discharged on the surface, ii) that even if they receive 2 electrons from the electrode, the concomitant absorption of the anions and donation of electron to the electrode decrease the electrosorption valence or iii) more likely, both things occur at the same time. To our knowledge, $\mathrm{z}_{\mathrm{u}}$ was never calculated in similar conditions to this paper. However, in agreement with this work, Deakin and Merloy ${ }^{44}$ found $\mathrm{z}_{\mathrm{u}} \approx 1.4$. However, it is worth noticing that the results were obtained at relatively high scan rates $\left(10 \mathrm{mV} . \mathrm{s}^{-1}\right)$ and more importantly, the Au electrode was obtained by sputtering and the CVs show a clear preferential orientation, resembling results obtained with $\mathrm{Au}(111)$. In fact, for the $\mathrm{Cu}$ UPD on $\mathrm{Au}(111)$, Legault et al. ${ }^{45}$ reported values of $\mathrm{Cu}$ electrosorption valence of $1.5-2$, while Omar et al. ${ }^{28}$ reported values around 1.

Concerning the UPD standard potential, we found similar values of $E_{\mathrm{Cu}^{2+} / \mathrm{Cu}(\mathrm{Au})}^{0}$ for both electrolytes, around $0.65 \pm 0.02 \mathrm{~V}$.

Having $E_{C u^{2+} / C u}^{0}$ and $E_{C u^{2+} / C u(A u)}^{0}$, we used equation 7 to calculate $\triangle E_{U P D}^{0}$. Table 2 shows that it is constant for the 3 systems evaluated in this work.

Table 2. $\triangle E_{U P D}^{0}$ for each electrolyte concentration.

\begin{tabular}{|c|c|c|}
\hline $\begin{array}{c}\Delta E_{U P D}^{\mathbf{0}} \text { for } \\
\mathrm{H}_{2} \mathrm{SO}_{4} \mathrm{0.05} \\
\mathbf{m o l} / \mathrm{kg}(\mathrm{V})\end{array}$ & $\begin{array}{c}\Delta E_{U P D}^{\mathbf{0}} \text { for } \\
\mathrm{H}_{2} \mathrm{SO}_{4} \mathrm{O.5} \\
\mathrm{mol} / \mathrm{kg}(\mathrm{V})\end{array}$ & $\begin{array}{c}\Delta E_{U P D}^{\mathbf{0}} \text { for } \\
\mathrm{HClO}_{4} \mathrm{0.05} \\
\mathrm{mol} / \mathrm{kg}(\mathrm{V})\end{array}$ \\
\hline $0.31 \pm 0.02$ & $0.31 \pm 0.02$ & $0.30 \pm 0.01$ \\
\hline
\end{tabular}


To obtain more $E_{M^{n+} / M(S)}^{0}$ values, some results can be extracted from the literature. For instance, the group of Itaya ${ }^{46}$ studied the UPD of $\mathrm{Cu}$ on $\mathrm{Au}(111)$ in $\mathrm{H}_{2} \mathrm{SO}_{4} 0.05$ mol. $\mathrm{kg}^{-1}$. As we calculated $a_{\mathrm{Cu}^{2+}}$ in this condition, by using the $\mathrm{CV}$ from their work, we found $E_{C u^{2+} / C u(A u(111))}^{0}=0.635$ and $\mathrm{z}_{\mathrm{u}}=1.3$ (Figure S4). The value for $E_{C u^{2+} / C u(A u(111) \text { ) }}^{0}$ is within the errors from our results with polycrystalline electrodes and for $\mathrm{z}_{\mathrm{u}}$ is very close. This shows the method easy applicability for a wide range of electrochemical data.

\section{Discussion}

In this paper, with the aim of finding a reliable parameter to characterize the UPD phenomenon, we started by writing the Nernst equation for the bulk deposition of metallic atoms and for UPD of the same atom on a metallic surface. Then, we showed how to straightforwardly calculate the standard potential for the UPD $\left(E_{M^{n+} / M(S)}^{0}\right)$, and, by comparison with the well-known potential for bulk deposition, we came up with a characteristic thermodynamic quantity of a given ion/substrate pair that we named "Standard UPD shift", $\triangle E_{U P D}^{0}$.

In contrast to the usually defined $\triangle E^{U P D}$, which has been determined at arbitrarily chosen electrolytes compositions, temperatures, scan rates, potential steps, etc., the $\triangle E_{U P D}^{0}$ is a far more fundamental property.

It is worth noting that, while $\triangle E^{U P D}$ measures the difference between the Gibbs free energy for the bulk deposition of an atom and the Gibbs free energy for the UPD of a monolayer of the same atom on a foreign substrate (experimentally the desorption process is measured), $\triangle E_{U P D}^{0}$ measures the difference between the Gibbs free energy for the bulk deposition of an atom and the Gibbs free energy for the UPD of a monolayer of the same atom on a foreign substrate in standard conditions $\left(\mathrm{T}=298 \mathrm{~K}, \mathrm{P}=1\right.$ bar and $\left.a_{M^{+n}}=1\right)$ by the extrapolation of the behaviour at zero coverage to a 1 monolayer.

To mathematically describe an UPD system from the onset of the deposition until the bulk deposition is likely impossible due to the myriad of parameters changing during the process (lateral interaction between the deposited adatoms, adsorption of ions, changes in the solvent configuration at the electric double layer, formation of complex mono-multilayers ${ }^{32}$, etc.). Thus, as usually done for electrolyte solutions, we characterize our real system by its departure from the ideally dilute solution. In this condition, we avoided several complexities described before:

i) We worked with low coverages, minimizing the interaction between the foreign atoms. 
ii) We fitted the results until $0.05 \mathrm{~V}-0.1 \mathrm{~V}$ after the onset of the UPD. This relatively short potentials range minimize the errors due to changes in the structure of the electric double layer and $2 \mathrm{D}$ transition phases.

We believe that this proof-of-concept paper brings promising results from several points of view. The experimental procedures and data treatment are straightforward, and values of $E_{M^{n+} / M}^{0}$ for several atoms are available in the literature in the so-called electrochemical series tables $^{47}$. As shown in this paper, it is possible to easily calculate the activity of the ions for the UPD in any system to obtain the desired standard parameters. Another important aspect is that we determined both $\triangle E_{U P D}^{0}$ and $E_{C u^{2+} / C u(A u)}^{0}$ in solutions of $\mathrm{H}_{2} \mathrm{SO}_{4}$ and $\mathrm{HClO}_{4}$, i.e., electrolytes with anions that show strong and weak adsorption, respectively. Besides, we have used solutions with acid concentrations differing by one order of magnitude and also obtained a result with a single crystal surface. Despite the fact that these huge differences are known to severely affect the UPD behaviour of the system $\mathrm{Cu}^{+2} / \mathrm{Au}^{36-38}$, we have surprisingly obtained constant values for $\triangle E_{U P D}^{0}$ and $E_{C u^{2+} / C u(A u)}^{0}$. More experiments are necessary to understand these results from a microscopic point of view.

We aim to use our method to determine $\Delta E_{U P D}^{0}$ and $E_{M^{n+} / M(S)}^{0}$ values for other UPD systems (including single crystals electrodes, as we did in this work using results from the literature) to offer reliable thermodynamic information which can be used as reference values for both experimental and theoretical UPD studies. In this sense, we believe that our parameters could certainly contribute with the development of works like that of Dabo et al. ${ }^{20}$, that would indeed contribute with the fundamental understanding of the UPD phenomenon.

\section{Conclusions}

Using cyclic voltammetry and open circuit potential measurements, performed in solutions containing different amounts of $\mathrm{Cu}^{2+}$ ions, plus a pure Nernstian treatment of UPD, we showed how to obtain $\triangle E_{U P D}^{0}$ and $E_{M^{n+} / M(S)}^{0}$, which are fundamental thermodynamic properties that permit the characterization of any UPD system.

We define a new thermodynamic parameter $\left(E_{M^{n+} / M(S)}^{0}\right)$, the standard potential of UPD. This quantity is the potential for the equilibrium between an atom $\mathrm{M}$ deposited on a substrate $S$ and ions $M^{n+}$ when $a_{M^{n+}}=1$ and $\theta_{M}=1$ and pretending ideally dilute behaviour on both, the solution and on the surface. 
We found that $\triangle E_{U P D, C u^{2+} / A u}^{0}=0.31 \mathrm{~V}, E_{C u^{2+} / C u(A u)}^{0}=0.65 \mathrm{~V}$ and that these quantities do not change with the activities of $\mathrm{Cu}^{2+}$ (which is desirable for a fundamental thermodynamic parameter) and with the nature and concentration of the electrolyte. Besides, surprisingly we found the same value for $E_{C u^{2+} / C u(A u(111))}^{0}$.

We aim to extend this result to other systems to contribute with a deeper understanding of the UPD phenomenon and bring new valuable information for both electrochemical and computational experiments.

\section{Acknowledgments}

PSF thanks Fundação de Amparo à Pesquisa do Estado de São Paulo (FAPESP) (grants: 2017/11986-5, 2018/20952-0, 2019/07449-0). PSF thanks Shell and the strategic importance of the support given by ANP (Brazil's National Oil, Natural Gas and Biofuels Agency) through the R\&D levy regulation. RAV thanks SAE-Unicamp, FAPESP (grants: 2018/132627 and 2019/13888-6) and CNPQ (grant: 136436/2019-6) for his scholarships.

\section{References}

1 S. Uhm, S. T. Chung and J. Lee, Electrochem. commun., 2007, 9, 2027-2031.

2 H. I. Karan, K. Sasaki, K. Kuttiyiel, C. A. Farberow, M. Mavrikakis and R. R. Adzic, ACS Catal., 2012, 2, 817-824.

3 D. Martín-Yerga, M. B. González-García and A. Costa-García, Sensors Actuators, B Chem., 2012, 165, 143-150.

4 Y. Dai and C. C. Liu, Sensors (Switzerland), , DOI:10.3390/s17050950.

5 C. R. Chenthamarakshan, Y. Ming and K. Rajeshwar, Chem. Mater, 2000, 12, 35383540 .

6 M. F. Cabral, M. L. Calegaro and S. A. S. MacHado, RSC Adv., 2012, 2, 2498-2503.

7 M. Alanyalioğlu, F. Bayrakçeken and Ü. Demir, Electrochim. Acta, 2009, 54, 6554-6559.

8 J. Horkans, I. H. Chang, P. C. Andricacos and H. Deligianni, J. Electrochem. Soc., 2019, 142, 2244-2249.

9 T. Binninger, E. Fabbri, R. Kötz and T. J. Schmidt, J. Electrochem. Soc., 2014, 161, H121-H128.

10 J. Solla-Gullón, P. Rodríguez, E. Herrero, A. Aldaz and J. M. Feliu, Phys. Chem. Chem. Phys., 2008, 10, 1359-1373.

11 C. H. Chen, D. Wu, Z. Li, R. Zhang, C. G. Kuai, X. R. Zhao, C. K. Dong, S. Z. Qiao, H. Liu and X. W. Du, Adv. Energy Mater., , DOI:10.1002/aenm.201803913.

12 O. A. Oviedo, L. Reinaudi, S. Garcia and E. P. M. Leiva, Underpotential Deposition, 2016.

13 S. R. Brankovic, J. X. Wang and R. R. Adžić, Surf. Sci., 2001, 474, L173-L179. 
F. Scholz and E. P. M. Leiva, ChemElectroChem, 2018, 5, 849-854.

S. E. Weitzner and I. Dabo, npj Comput. Mater., 2017, 3, 0-1. 1894.

D. Fraenkel, J. Phys. Chem. B, 2012, 116, 11662-11677.

J. Torrent, F. Sanz and J. Virgili, J. Solution Chem., 1986, 15, 363-375.

J. Clavilier, R. Faure, G. Guinet and R. Durand, J. Electroanal. Chem., 1980, 107, 205209.

25 I. Mills, IUPAC gren book, 1993.

I. Langmuir, J. Am. Chem. Soc., 1918, 40, 1361-1403.

27 P. Vanýsek, Handb. Chem. Phys., 2012, 93, 5-80.

28 I. H. Omar, H. J. Pauling and K. Jüttner, J. Electrochem. Soc., 2019, 140, 2187-2192.

29 J. W. Schultze and D. Dickertmann, Surf. Sci., 1976, 54, 489-505.

30 L. Blum, H. D. Abruña, J. White, J. G. Gordon, G. L. Borges, M. G. Samant and O. R. Melroy, J. Chem. Phys., 1986, 85, 6732-6738.

31 O. R. Melroy, M. G. Samant, G. L. Borges, J. G. Gordon, L. Blum, J. H. White, M. J. Albarelli, M. McMillan and H. D. Abruna, Langmuir, 1988, 4, 728-732.

B. Madry, K. Wandelt and M. Nowicki, Electrochim. Acta, 2016, 217, 249-261. N. Vasiljevic, L. T. Viyannalage, N. Dimitrov and K. Sieradzki, J. Electroanal. Chem., 2008, 613, 118-124.

M. S. Zei, G. Qiao, G. Lehmpfuhl and D. M. Kolb, Berichte der Bunsengesellschaft für Phys. Chemie, 1987, 91, 349-353.

J. Hotlos, O. M. Magnussen and R. J. Behm, Surf. Sci., 1995, 335, 129-144.

S. Frittmann and R. Schuster, J. Phys. Chem. C, 2016, 120, 21522-21535.

38 N. Markovic and P. N. Ross, Langmuir, 1993, 9, 580-590.

39 V. Climent and J. M. Feliu, J. Solid State Electrochem., 2011, 15, 1297-1315.

40 E. Avci, Freie Universität Berlin, 2007.

41 A. J. deBethune, T. S. Licht and N. Swendeman, J. Electrochem. Soc., 1959, 106, 626627.

42 W. E. Tragert and W. D. Robertson, J. Electrochem. Soc., 1955, 102, 86.

43 J. Bard, A. J., Parsons, R., Jordan, Standard Potentials in Aqueous Solutions, Marcel Dekker, INC., New York, 1985.

44 M. R. Deakin and O. Melroy, J. Electroanal. Chem., 1988, 239, 321-331.

45 M. Legault, L. Blum and D. A. Huckaby, J. Electroanal. Chem., 1996, 409, 79-86.

46 T. Hachiya, H. Honbo and K. Itaya, J. Electroanal. Chem., 1991, 315, 275-291.

47 P. Vanysek, CRC Handb. Chem. Phys. 


\section{Figures}
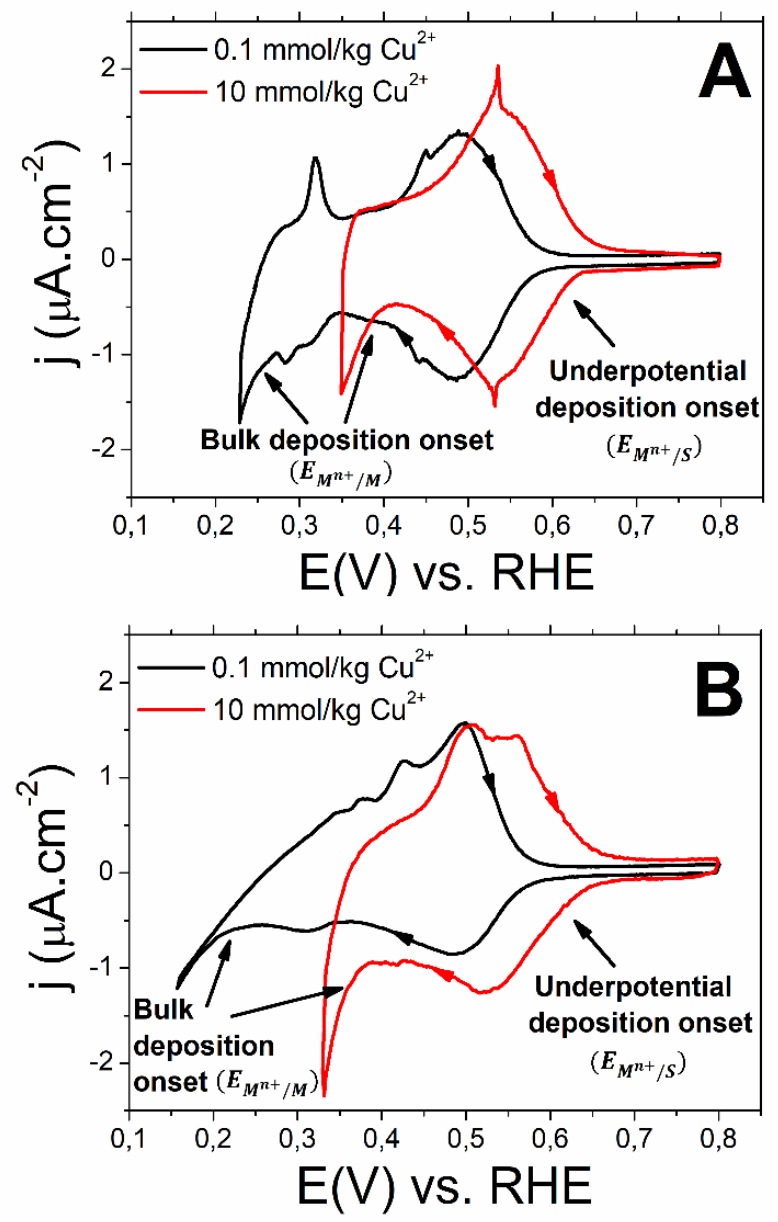

Figure 1. UPD profile for the deposition of: A) 0.1 and $10 \mathrm{mmol} / \mathrm{kg}$ of $\mathrm{Cu}^{2+}$ on $\mathrm{Au}_{\mathrm{p}}$ in $\mathrm{H}_{2} \mathrm{SO}_{4}$ $0.5 \mathrm{~mol} / \mathrm{kg}$. B) 0.1 and $10 \mathrm{mmol} / \mathrm{kg}$ of $\mathrm{Cu}^{2+}$ on $\mathrm{Au}_{\mathrm{p}}$ in $\mathrm{HClO}_{4} 0.5 \mathrm{~mol} / \mathrm{kg}$. Sweep rate $=1 \mathrm{mVs}^{-}$ 1. The onset for the upd and bulk deposition regions described on equations 1 and 2 are highlighted.

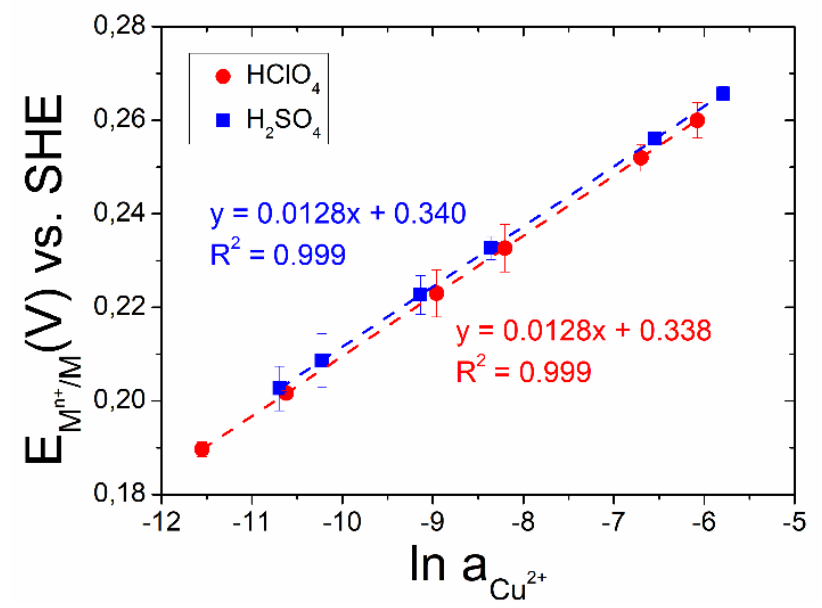


Figure 2. $E_{\mathrm{Cu}^{2+} / \mathrm{Cu}}$ determined by $\mathrm{OCP}$ in function of the activity of $\mathrm{Cu}^{2+}$ for $\mathrm{H}_{2} \mathrm{SO}_{4} .0 .05 \mathrm{~mol} / \mathrm{kg}$ and $\mathrm{HClO}_{4} 0.05 \mathrm{~mol} / \mathrm{kg}$.

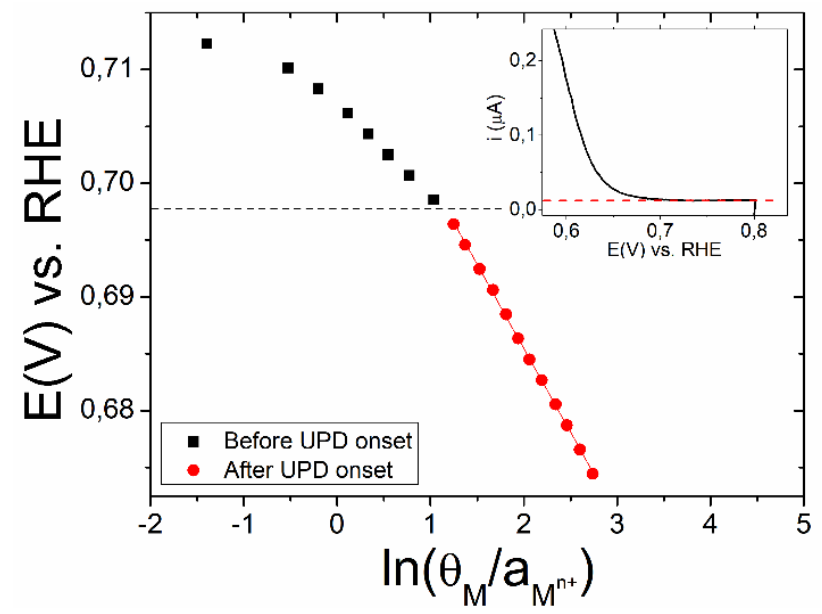

Figure 3. $E_{C u^{2+} / C u(A u)}$ vs. $\ln \left(\theta_{C u} / a_{C u^{2+}}\right)$ for the UPD of $\mathrm{Cu}$ on $\mathrm{Au}_{\mathrm{p}}$ in $\mathrm{H}_{2} \mathrm{SO}_{4} 0.05 \mathrm{~mol} / \mathrm{kg}$ and $\mathrm{Cu}^{2+} 1 \mathrm{mmol} / \mathrm{kg}$. Inset: cyclic voltammetry for the same conditions. We considered a constant current for the electric double layer. This current (red dashed line) was subtracted from that of the cyclic voltammetry. 